\title{
Comparative Analysis of Linear and Nonlinear Pattern Synthesis of Hemispherical Antenna Array Using Adaptive Evolutionary Techniques
}

\author{
K. R. Subhashini and A. T. Praveen Kumar \\ Department of Electrical Engineering, National Institute of Technology, Rourkela 769008, India \\ Correspondence should be addressed to K. R. Subhashini; subppy@gmail.com
}

Received 12 September 2013; Revised 8 November 2013; Accepted 8 November 2013; Published 22 January 2014

Academic Editor: Hon Tat Hui

Copyright (C) 2014 K. R. Subhashini and A. T. P. Kumar. This is an open access article distributed under the Creative Commons Attribution License, which permits unrestricted use, distribution, and reproduction in any medium, provided the original work is properly cited.

\begin{abstract}
Hemispherical antenna arrays are subjected to linear and nonlinear synthesis and are optimized using adaptive based differential evolution (ADE) and fire fly (AFA) algorithm. The hemispherical shaped array with isotropic elements is considered. Antenna element parameters that are used for synthesis are excitation amplitude and angular position. Linear synthesis is termed as the variation in the element excitation amplitude and nonlinear synthesis is process of variation in element angular position. Both ADE and AFA are a high-performance stochastic evolutionary algorithm used to solve $N$-dimensional problems. These methods are used to determine a set of parameters of antenna elements that provide the desired radiation pattern. The effectiveness of the algorithms for the design of conformal antenna array is shown by means of numerical results. Comparison with other methods is made whenever possible. The results reveal that nonlinear synthesis, aided by the discussed techniques, provides considerable enhancements compared to linear synthesis.
\end{abstract}

\section{Introduction}

Design of antenna arrays that conforms an arbitrary shape has triggered the electromagnetic researchers since they have a potential of $360^{\circ}$ coverage, either with an omnidirectional beam, multiple beams, or a narrow beam that can be steered over $360^{\circ}$. One of the geometrical solutions that can be provided through a hemispherical shape. The linear arrays have good directivity and they can form the narrowest main lobe in a given direction but cannot be steered in all azimuth directions. The circular array is a high side lobe geometry. The lacuna of the regular arrays leads to the aid of arrays that can perform/radiate in azimuth range also [1]. These arrays have to be mathematically formulated for their array factor. Since the array elements are conformal to the surface of the sphere, the phase at each element becomes critical for synthesizing low side lobe patterns [2, 3]. Primitive methods such as Schelkunoff, Fourier, and Woodwardlawson cannot be applied for this hemispherical array because of its curvature effects; the phase adjustments of each array element become complex. The stochastic pattern synthesis [4-7] can be adopted for these conformal shapes. The literature instructs the advantages of stochastic process over deterministic methods that can be stretched out for large size arrays [8-11]. One of the greatest advantages of using these evolutionary techniques such as Genetic Algorithm and Particle Swarm optimization [12-14] is their ability to deal with a huge parameter set up with out getting trapped in the local minima and with the advent of high computing facility these can be easily simulated [7, 15-18]. Recently invented high performance algorithm is Fire fly algorithm. DE involves mutation and crossover strategies that influence the results. Usage of the DE in the design of antenna array is available. Chen et al. [19] proposed antenna array optimization for various pattern synthesis objectives. Mandal et al. [20-24] have proposed many modified versions to the standard DE algorithm. On the other hand the literature on FA method in the design of antenna arrays is not extensive, a sample of which can be found in [25-28]. In this paper, the method of FA is used to provide a comprehensive study of the design 
of a mathematically modelled hemispherical antenna array. The parameters of antenna elements that provide the goal radiation pattern are optimized using both DE and FA. The effectiveness of these methods for the design of antenna arrays is shown by means of numerical results. The results reveal that design of hemispherical antenna array antenna and using these techniques to optimize element excitation and angular positioning of the elements provide considerable improvement in the directivity and a sharper beam widths.

\section{Formulation of Array Factor for a Hemispherical Shaped Antenna Array}

In this geometry the antenna elements are placed in the combination of linear and circular arrays. Hence the array factor equations of linear and circular array are utilized to deduce the array factor equation of the hemispherical arrangement see Figure 1. The array factor equation for $M$ element linear array with spacing " $d$ " is

$$
(\mathrm{AF})_{M}(\theta, \phi)=\sum_{m=1}^{M} e^{j(m-1) k d \cos \theta+\beta}
$$

And each circular arrangement with uniform spacing containing " $N$ " elements is given as

$$
(\mathrm{AF})_{N}(\theta, \phi)=\sum_{n=1}^{N} I_{n} e^{j\left(k a_{m} \sin \theta \cos \left(\phi-\phi_{n}\right)+\alpha_{n}\right)}
$$

The stack of elements is not considered at the apex of the sphere. Consider the following:

$$
\begin{aligned}
& a_{m}=r_{d} \sin \theta_{m}, \\
& d_{m}=r_{d} \cos \theta_{m}, \\
& \sqrt{a_{m}^{2}+d_{m}^{2}}=r_{d}, \\
& a_{m}^{2}=\sqrt{r_{d}^{2}-d_{m}^{2}} .
\end{aligned}
$$

The angular spacing between two elements in the circular arrangement is given by

$$
\begin{aligned}
& \sin \phi_{n}=\frac{d_{m}}{a_{m}}, \\
& \sin \left(\frac{2 \pi}{N}\right)=\frac{d_{m}}{a_{m}}, \\
& d_{m}=a_{m} \sin \left(\frac{2 \pi}{N}\right) .
\end{aligned}
$$

In (2) $a_{m}$ is the radius of $m$ th circle. And the assumption is made such that radius of $a_{m}<a_{0}$, where $a_{0}$ is the radius of the initial circle in $X-Y$ plane. The linear distance between two circular stacks is same as the distance between two linear ments, that is, " $d$ ". In order to steer beam towards $\left(\theta_{s}, \phi_{s}\right)$ direction the progressive phase shift $\alpha_{n}$ has to be maintained as

$$
\alpha_{n}=-j k a_{m} \sin \theta_{s} \cos \left(\phi_{s}-\phi_{n}\right)
$$

Hence the array factor of the hemispherical arrangement of elements is framed as

$$
\begin{aligned}
& (\mathrm{AF})_{\text {hemi }}(\theta, \phi) \\
& \quad=\sum_{m=1}^{M} I_{m} e^{j\left(k d_{m} \cos \theta+\beta\right)} \cdot\left[\sum_{n=1}^{N} I_{n} e^{-j k a_{m} \sin \theta \cos \left(\phi-\phi_{n}\right)+\alpha_{n}}\right],
\end{aligned}
$$

where $d_{m}=(m-1) d$.

\section{Overview of DE and FA Algorithms}

3.1. Differential Evolution. Evolutionary algorithms (EAs) are population-based stochastic algorithms that can effectively handle real-world optimization problems which are noncontinuous and/or nondifferentiable and characterized by chaotic disturbances, randomness, and complex nonlinear dynamics. DE is a Stochastic Direct Search and Global Optimization algorithm. It uses rather greedy selection and less stochastic approach to solve optimization problems when compared to other classical EAs. DE is an evolutionary computation method that was originally introduced by Storn and Price in 1995. Furthermore, it has been modified by researchers [29] to be a reliable and versatile function optimizer that is also readily applicable to a wide range of optimization problems. Mutation and crossover strategies along with their associated parameters show the effectiveness of the conventional DE in solving numerical optimization problems. DE has the ability to find the global minimum regardless of the initial parameter values and can give reasonably fast convergence see Figure 2 .

DE uses a population of size $P$, composed of floating point encoded individuals that evolve over generations $G$ to reach an optimal solution. Each $W o$ is a vector that contains as many parameters as the problem decision variables $D$. The population size $P$ is an algorithm control parameter selected by the user which remains constant throughout the optimization process. With the present weight current fitness is found and compared with the new fitness value, the weights will be updated by employing mutation $F$ and crossover CR. Pseudo code of the algorithm presented in Pseudocode 1.

3.2. Firefly Algorithm. Firefly algorithm was developed by Xin-She Yang at Cambridge university in 2007. It uses the following two idealized rules: (1) all fireflies are unisex so that a firefly will be attracted to other fireflies regardless of their sex; (2) attractiveness is proportional to their brightness and they both vary with the distance between them. FA initially produces a swarm of fireflies located randomly in the search space. The initial distribution is usually produced from a uniform random distribution. The position of each firefly in the search space represents a potential solution of the optimization problem. The dimension of the search space is equal to the number of optimizing parameters in the given problem. The fitness function takes the position of a firefly as input and produces a single numerical output value denoting how good the potential solution is. A fitness value is assigned to each firefly. The FA uses a phenomenon known is bioluminescent communication to model the movement 


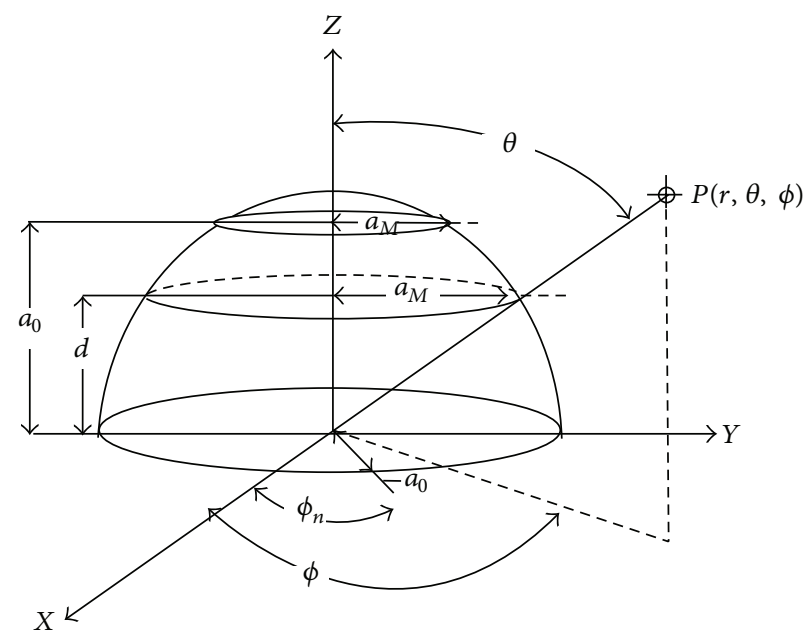

Figure 1: Geometry of the hemisphere.

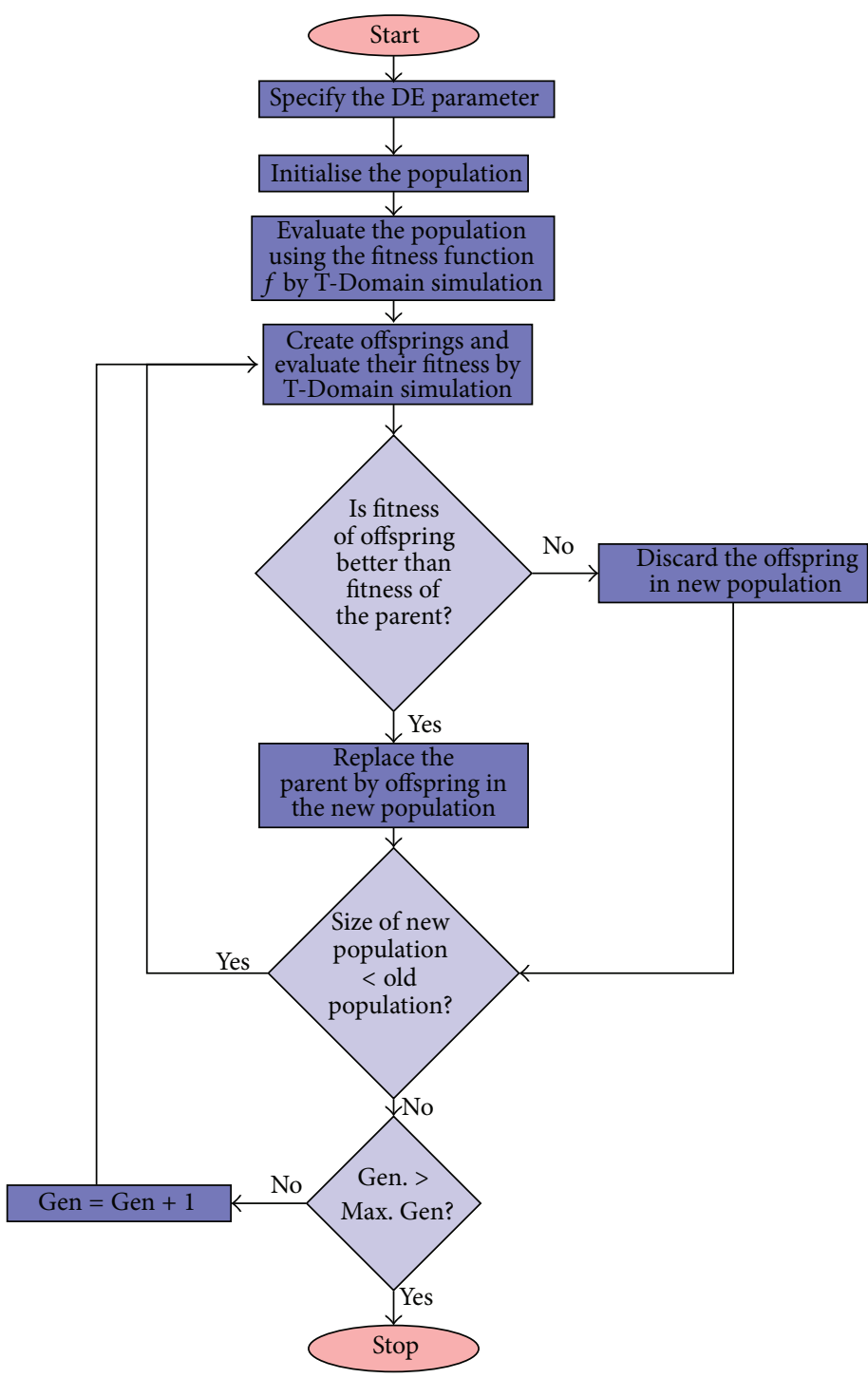

FIgURE 2: Flow Chart for differential evolution algorithm. 


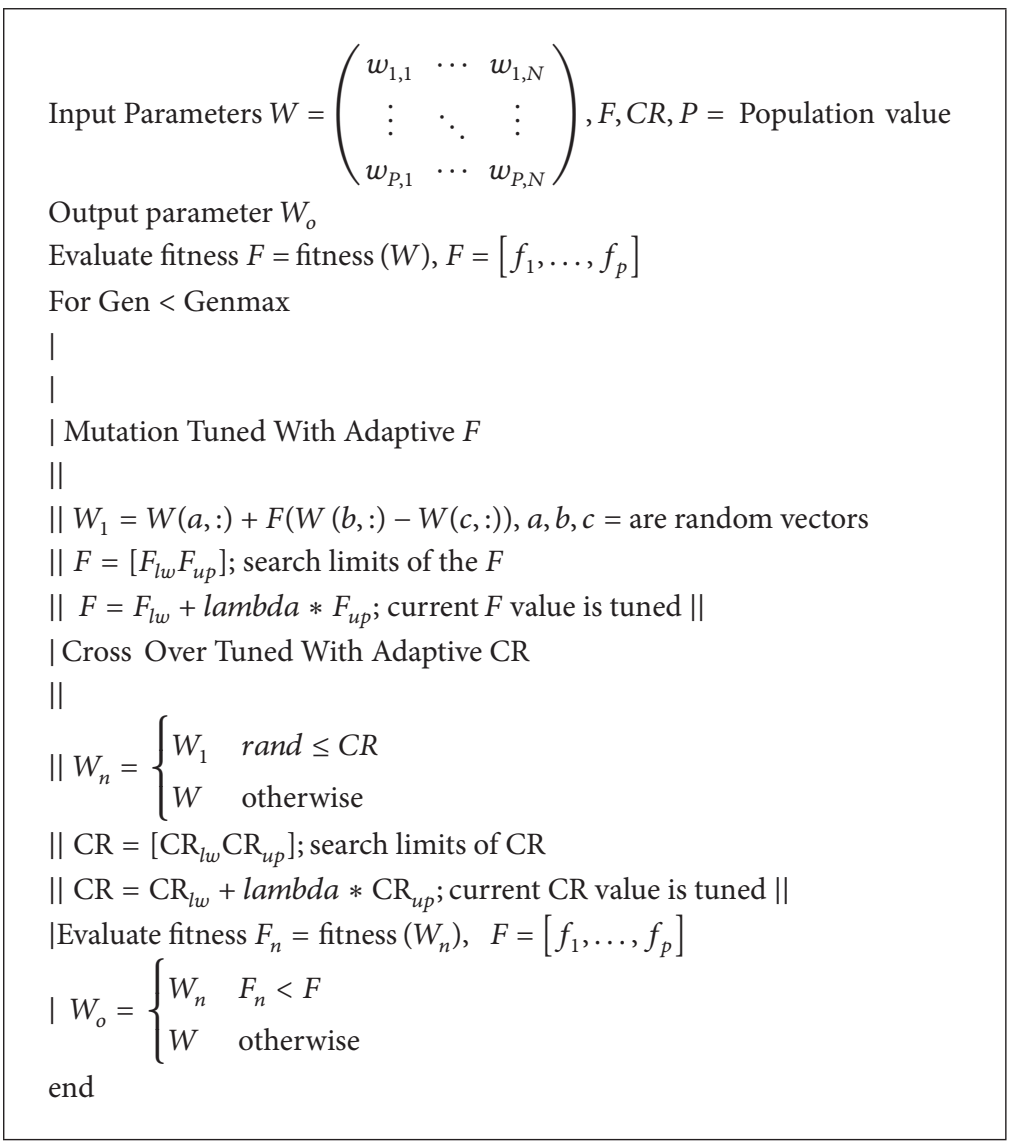

Pseudocode 1: Pseudocode of Adaptive DE Algorithm.

of the fireflies through the search space. The brightness of each firefly depends on the fitness value of that firefly. Each firefly is attracted by the brightness of other fireflies and tries to move towards them. The velocity or the pull of a firefly towards another firefly depends on the attractiveness. The attractiveness depends on the relative distance between the fireflies. It can be a function of the brightness of the fireflies as well. A brighter firefly that is far away may not be as attractive as a less bright firefly that is closer. In each iterative step, FA computes the brightness and the relative attractiveness of each firefly. Depending on these values, the positions of the fireflies are updated [25-28]. After a sufficient amount of iterations, all fireflies converge to the best possible position on the search space. The antenna problems are addressed by this FA algorithm in the literature in recent times and have not been properly explored with the updated equations. An attempt is made in this paper to explore, modify, and apply this FA to antenna pattern synthesis see Figure 7 and Pseudocode 2.

\section{Minimize the Maximum Side Lobe Ratio, Null Detection, and Beam Width}

The DE and FA algorithms are used to obtain the optimal synthesis of a $M * N$ hemispherical array in order to minimize the SLL ratio range in a specified region and to detect null points and beam width within the decided angle. Consider the following:

$$
\begin{aligned}
& o f_{1}=\frac{1}{\Delta \theta_{M L}} \int_{\theta_{M L L}}^{\theta_{M S U}}\left|\operatorname{AF}\left(\theta_{o}\right)-\operatorname{AF}\left(\theta_{d}\right)\right|^{2} d \theta, \\
& o f_{2}=\frac{1}{\Delta \theta_{i}} \int_{\theta_{s l i}}^{\theta_{s u i}}\left|\operatorname{AF}\left(\theta_{o}\right)-\operatorname{AF}\left(\theta_{d}\right)\right|^{2} d \theta, \\
& o f_{3}=\frac{1}{\Delta \theta_{N i}} \int_{\theta_{N l i}}^{\theta_{N u i}}\left|\operatorname{AF}\left(\theta_{o}\right)-\operatorname{AF}\left(\theta_{d}\right)\right|^{2} d \theta,
\end{aligned}
$$

where $\operatorname{AF}\left(\theta_{o}\right)$ is the obtained array factor and $\operatorname{AF}\left(\theta_{d}\right)$ is the desired array factor. The fitness is framed, taking into account the above discussed three constraints, and summarized as the objective function given in (8) is the weighted sum of the individual objectives. $k_{1}, k_{2} \in[0,1]$. Consider the following:

$$
\text { fitness }=k_{1} *\left(\frac{o f_{1}}{o f_{2}}\right)+k_{2} * o f_{3} \text {. }
$$




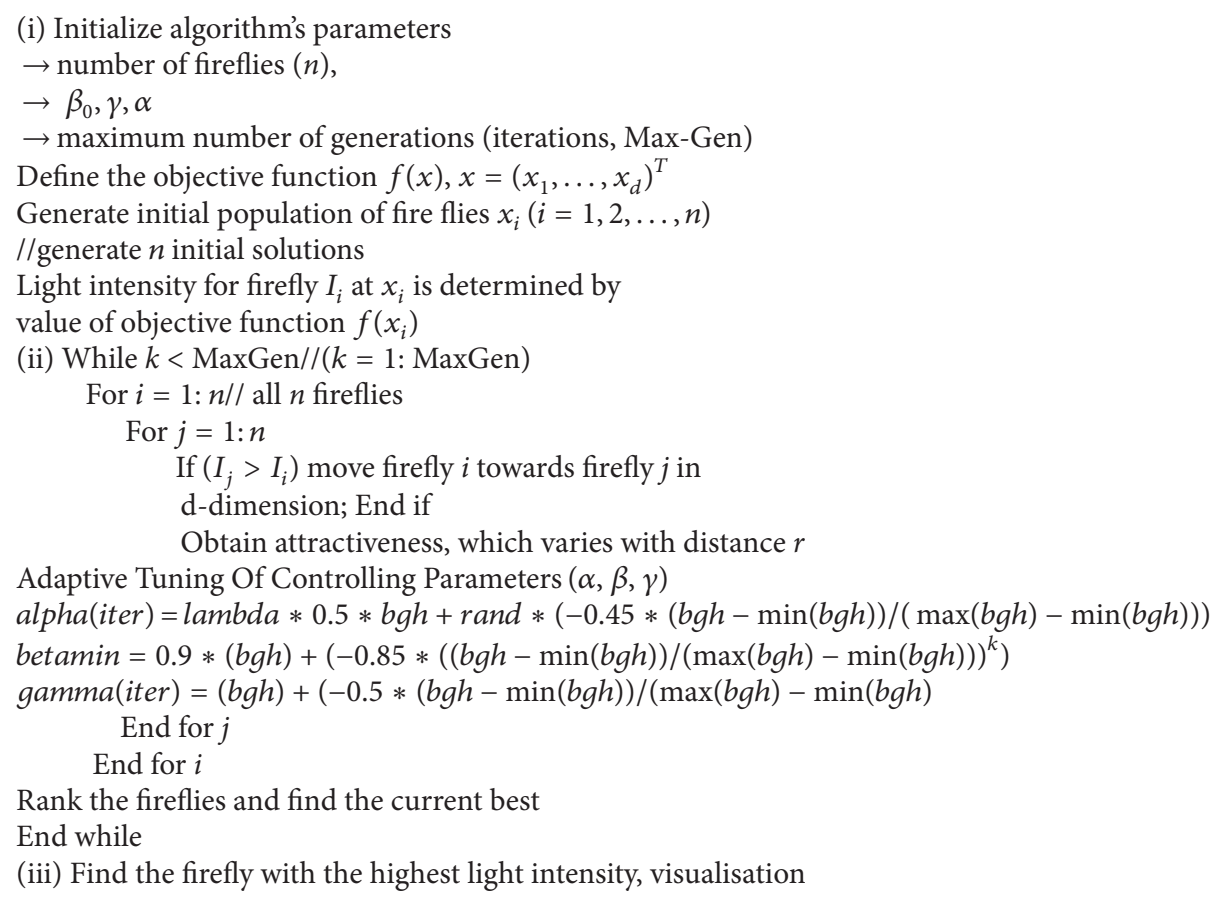

Pseudocode 2: Pseudocode of Adaptive Fire Fly Algorithm.

4.1. Optimize Elements Amplitudes $I_{m n}$ and Element Positions $\left(\theta_{P}, \phi_{\mathrm{Q}}, \alpha_{N M}, \phi_{N M}\right)$. Analytical synthesis approach is framed for the synthesis of multidimensional array geometry,

$$
\begin{aligned}
& {\left[\begin{array}{c}
\operatorname{AF}\left(\theta_{1}, \phi_{1}\right) \\
\operatorname{AF}\left(\theta_{1}, \phi_{2}\right) \\
\cdot \\
\operatorname{AF}\left(\theta_{2}, \phi_{1}\right) \\
\operatorname{AF}\left(\theta_{2}, \phi_{2}\right) \\
\cdot \\
\operatorname{AF}\left(\theta_{3}, \phi_{1}\right) \\
\operatorname{AF}\left(\theta_{3}, \phi_{2}\right) \\
\cdot \\
\operatorname{AF}\left(\theta_{P}, \phi_{\mathrm{Q}}\right)
\end{array}\right]} \\
& =\left[\begin{array}{ccc}
E\left(\theta_{1}, \phi_{1}, \alpha_{1}, \phi_{1}\right) & \cdot & F\left(\theta_{1}, \phi_{1}, \alpha_{N M}, \phi_{N M}\right) \\
E\left(\theta_{1}, \phi_{2}, \alpha_{1}, \phi_{1}\right) & \cdot & F\left(\theta_{1}, \phi_{2}, \alpha_{N M}, \phi_{N M}\right) \\
\ldots & \cdot & \\
E\left(\theta_{2}, \phi_{1}, \alpha_{1}, \phi_{1}\right) & \cdot & F\left(\theta_{2}, \phi_{1}, \alpha_{N M}, \phi_{N M}\right) \\
E\left(\theta_{2}, \phi_{1}, \alpha_{1}, \phi_{1}\right) & \cdot & F\left(\theta_{2}, \phi_{2}, \alpha_{N M}, \phi_{N M}\right) \\
\cdot & & \\
\cdot & \cdot & \\
E\left(\theta_{P}, \phi_{\mathrm{Q}}, \alpha_{1}, \phi_{1}\right) & \cdot & F\left(\theta_{P}, \phi_{\mathrm{Q}}, \alpha_{N M}, \phi_{N M}\right)
\end{array}\right] \\
& \times\left[\begin{array}{c}
I_{1} \\
I_{2} \\
\cdots \\
\cdot \\
I_{M N}
\end{array}\right] .
\end{aligned}
$$

The mathematical form of the above explained equation is where $\operatorname{AF}(\theta, \phi)=E(\theta, \phi)[I] .\left[\theta_{1} \theta_{2} \cdots \theta_{P}\right]$ represents the sample points of the elevation range. $\left[\phi_{1} \phi_{2} \cdots \phi_{\mathrm{Q}}\right]$ represents the sample points of the azimuthal range. $\left[I_{1} I_{2} \cdots I_{M}\right]$ represents the excitation current vector and $\left[\alpha_{1} \cdots \alpha_{N M}\right]$ and $\left[\phi_{1} \cdots \phi_{\mathrm{Q}}\right]$ its associated excitation angle vector and angular position of each current element over each circular stack which forms a conformal shape. The conformal linear synthesis points out to the variation of the matrix $F\left(I_{1} I_{2} \cdots I_{M N}\right)$. The conformal non linear synthesis points out to the variation of the matrix $F\left(\theta_{P}, \phi_{\mathrm{Q}}, \alpha_{N M}, \phi_{N M}\right)$. The amplitudes of the elements $I_{n m}$ are varied and angle $\theta$ of the main beam direction is kept constant; the angular distance between the elements is also kept constant. The initial values of the current amplitudes provided to the linear array are set to be uniformly distributed from $[0,1]$. Nonuniform synthesis can be achieved by adapting the angular positions of the elements for the objective function given by (8). Mathematically this non linear synthesis is adjusting the $F\left(\theta_{P}, \phi_{\mathrm{Q}}, \alpha_{N M}, \phi_{N M}\right)$ parameter by altering the depending values $\phi$ and $\alpha$ by maintaining the excitation amplitudes uniform.

The case study is performed on various array sizes starting with eight elements arranged in two circles with four elements each. The radiation pattern is shown in Figure 3. With this array size the assumed desired pattern is not attained, particularly the side lobe level region is at $-10 \mathrm{~dB}$. Both the optimization techniques in the uniform and nonuniform synthesis do not meet the objective. The array size is increased to sixteen elements which could show a small effect on the side lobe level but not considerate. This is shown in Figure 3(b). The cost function curve also settled at a high value is shown in Figures 4(a) and 4(b). The corresponding 


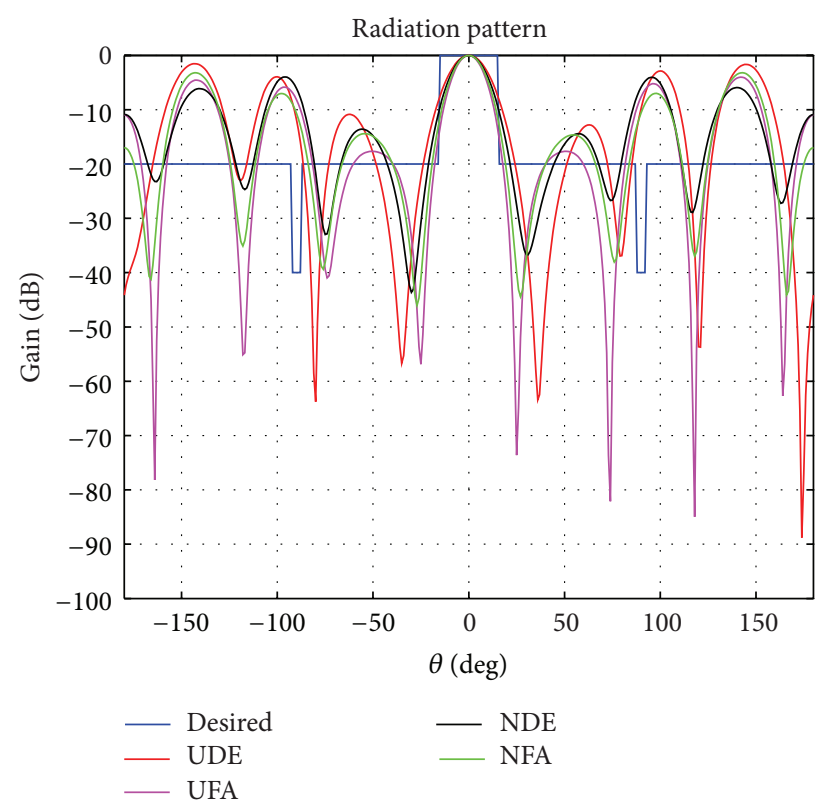

(a)

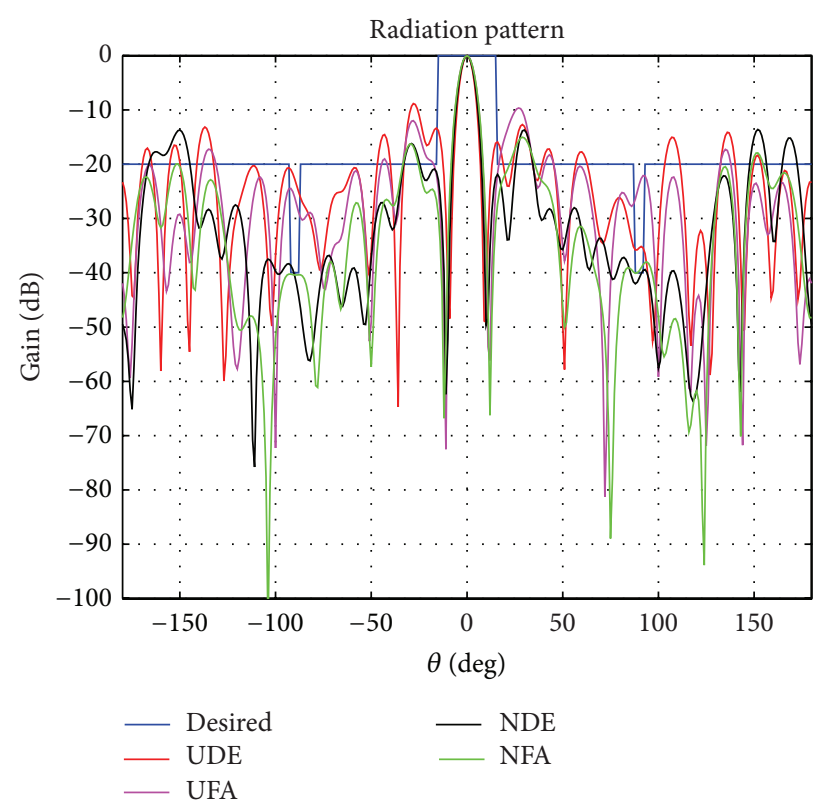

(b)

FIgURE 3: Radiation pattern for (a) $N \times M=2 \times 4$, (b) $N \times M=4 \times 4$.

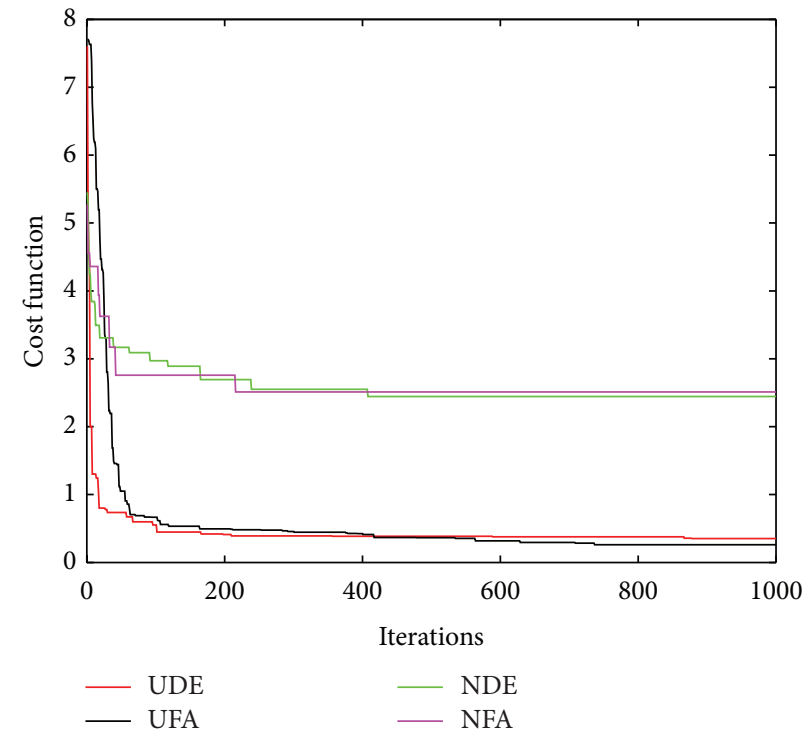

(a)

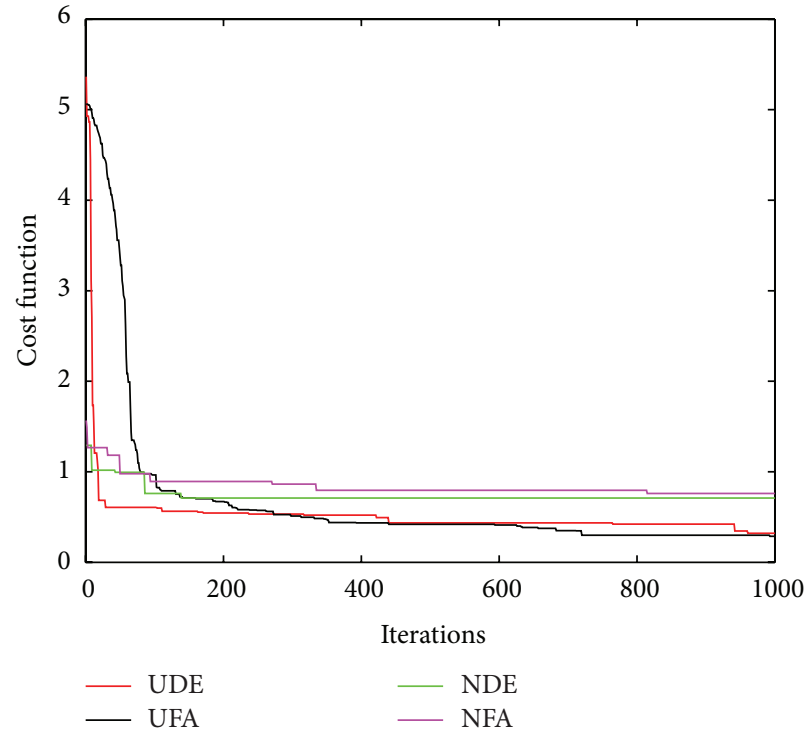

(b)

Figure 4: Fitness curves for (a) $N \times M=2 \times 4$, (b) $N \times M=4 \times 4$.

radiation pattern in the azimuth plane $(x-y)$ for amplitude and position optimization using DE and FA for various array sizes is shown in Figures 4 and 6 . Figure 5 shows the simulated result of twenty-five elements. The radiation pattern of the nonuniform synthesis using DE reflects a lower side lobe level, with a minimum cost function shown in Figure 6.

The array size is varied from eight elements (i.e., two concentric circles of four elements) to forty-nine elements. The amplitude optimization by DE and FA are labelled as
UDE and UFA. On the other hand position optimization by DE and FA are labelled as NDE and NFA. The corresponding radiation patterns are given in Figure 5. Pattern graphs reveal that for small array size position optimization is not desirable as the SLL has not settled at $-20 \mathrm{~dB}$. With the increase in the array size the optimized pattern of non linear synthesis adapted fast to the desired. Considering the algorithm point of view and the cost of evaluation for both uniform and nonuniform pattern syntheses adaptive DE comes out as a low cost value to produce the nearly optimum pattern. 


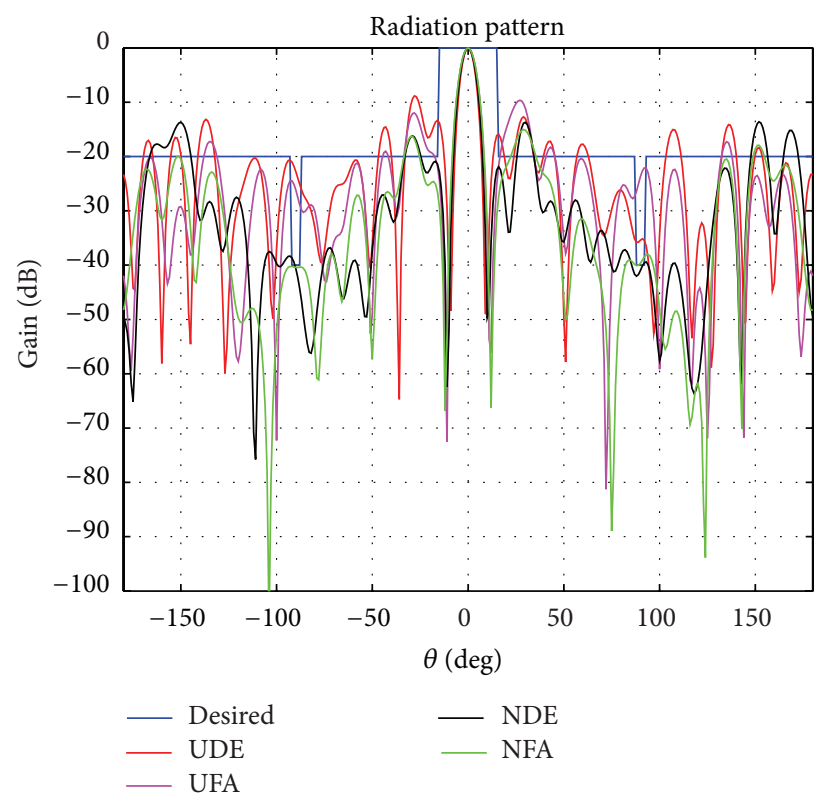

(a)

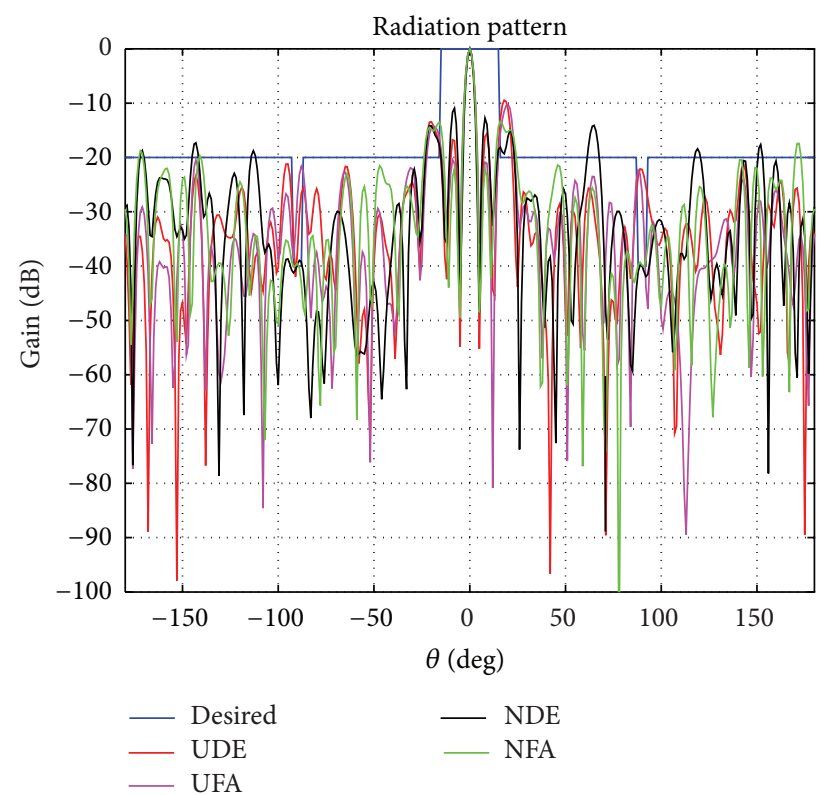

(b)

FIgURE 5: Radiation pattern for (a) $N \times M=5 \times 5$, (b) $N \times M=7 \times 7$.

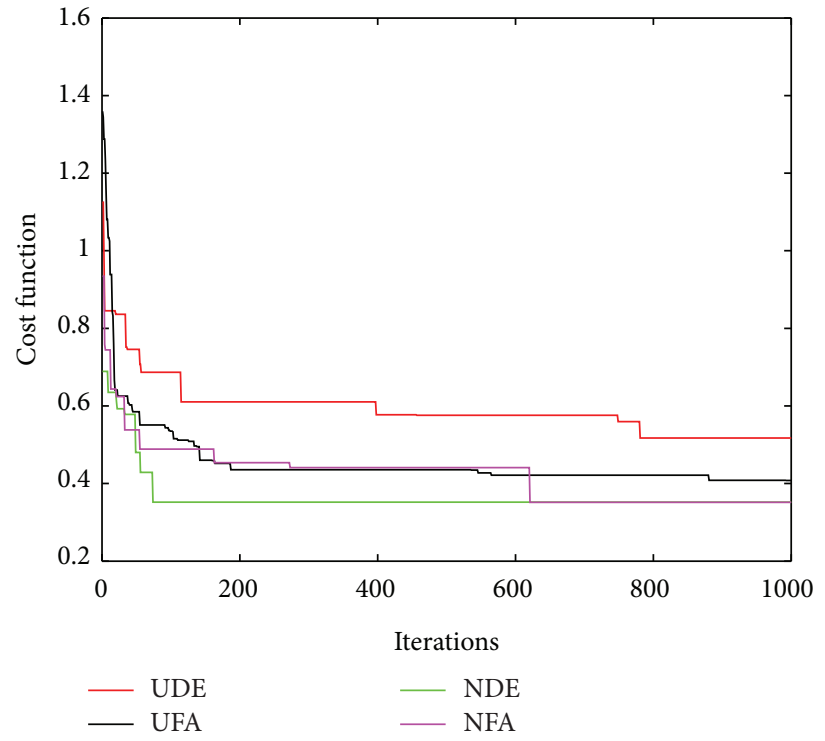

(a)

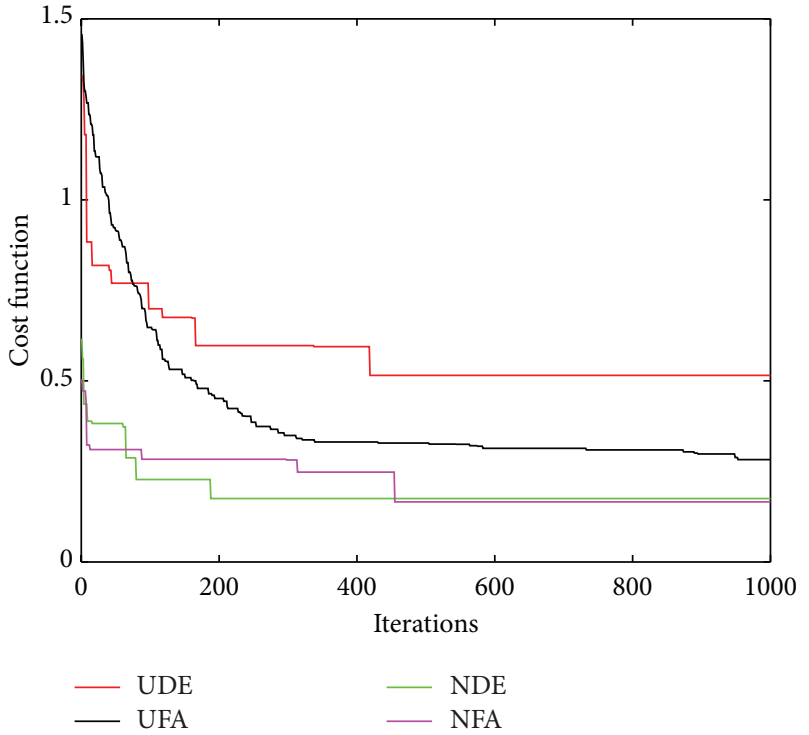

(b)

Figure 6: Fitness curves for (a) $N \times M=5 \times 5$, (b) $N \times M=7 \times 7$.

However the iterations were fixed to 1000 and evaluated for best 15 runs. If the iterations are increased the performance of the evolutionary fire fly can be further improved.

A statistical comparison of the antenna array performance values is tabulated in Table 1 . The numerical statistics show an enhanced directivity value of $29.1 \mathrm{~dB}$ for a nonuniform pattern synthesis using DE algorithm. The numericals listed out in the directivity column stand as an fetching property in choosing this geometry. Another achievement of this geometry is the sharper beam for large array sizes.

\section{Conclusion}

The paper concludes with the mathematical assumption and formulation of a hemispherical antenna array. A multiobjective function is chosen with different constraints. The 


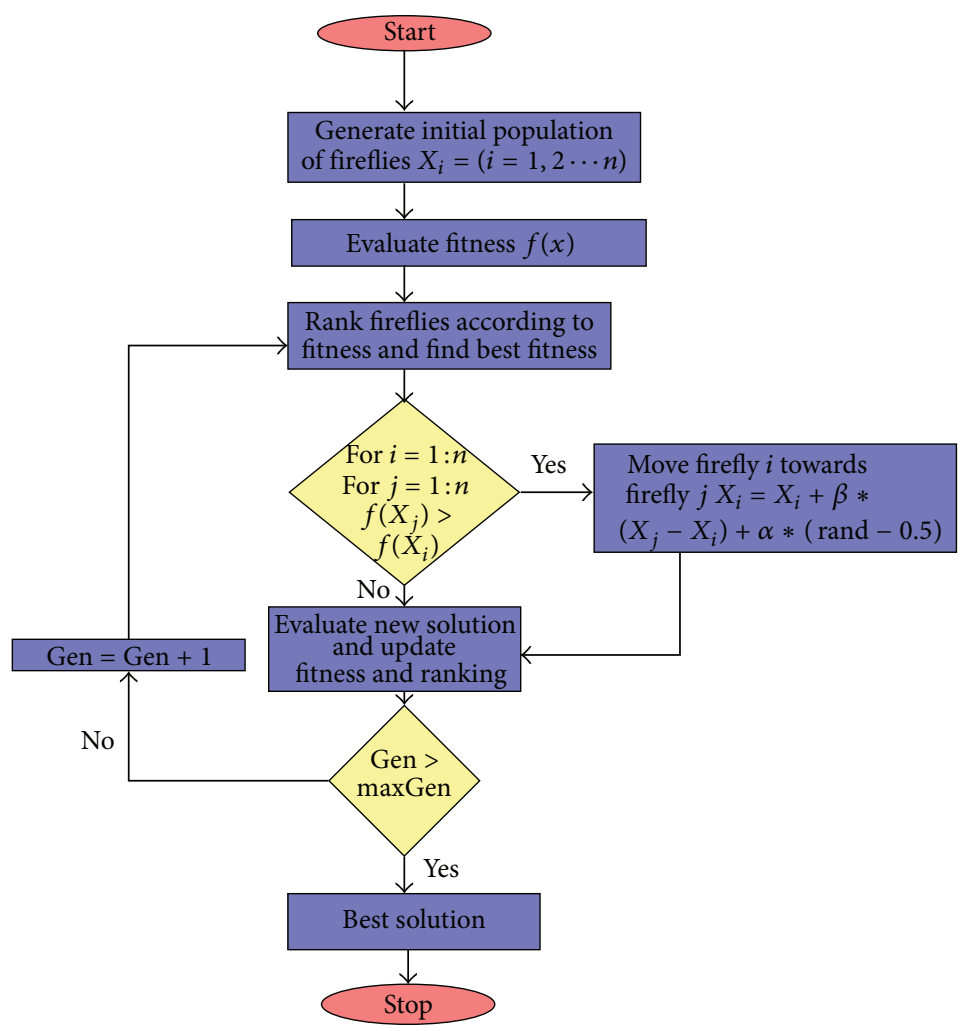

FIGURE 7: Flowchart of firefly algorithm.

TABLE 1: Statistical antenna array performance data of hemispherical nonuniform synthesis.

\begin{tabular}{|c|c|c|c|c|c|c|c|c|c|c|c|c|}
\hline \multirow{2}{*}{$\begin{array}{l}\text { Number of } \\
\text { El }\end{array}$} & \multicolumn{4}{|c|}{$\begin{array}{l}\text { Directivity } \\
\text { (DB) }\end{array}$} & \multicolumn{4}{|c|}{$\begin{array}{l}\text { Side lobe level } \\
\text { (DB) }\end{array}$} & \multicolumn{4}{|c|}{$\begin{array}{c}\text { HPBW } \\
\text { (degrees) }\end{array}$} \\
\hline & NDE & NFA & UDE & UFA & NDE & NFA & UDE & UFA & NDE & NFA & UDE & UFA \\
\hline $2 * 4$ & 14.4 & 14.5 & 13.1 & 12.9 & -1.38 & -20.84 & -9 & -0.5 & 16 & 16 & 18 & 16 \\
\hline $4 * 4$ & 20.1 & 21.3 & 16.5 & 16.2 & -13.72 & -18.89 & -20 & -20 & 10 & 10 & 10 & 10 \\
\hline $5 * 5$ & 23.9 & 24.1 & 22.3 & 22.7 & -13.48 & -10.21 & -7 & -8 & 6 & 6 & 7 & 7 \\
\hline $6 * 6$ & 26.8 & 26.6 & 25.1 & 25 & -18.73 & 13.44 & -15 & -19 & 4 & 4 & 3 & 3 \\
\hline $7 * 7$ & 29.1 & 28.7 & 25.5 & 26.1 & -21.11 & -14.67 & -14 & -11 & 4 & 4 & 3 & 3 \\
\hline
\end{tabular}

radiation pattern of this array is synthesized using two evolutionary based adaptive algorithms, where the excitations and angular positions are optimized and comparatively analysed. Nonuniform synthesis of hemispherical arrangement beats out the other conformal shapes in tracking applications because of the regularity and uniformity in the shape. The sharper beam width of $2^{\circ}$ has a good application in medical fields. Numerical results show that firefly and differential evolution compete with each other in satisfying the objectives. FA maintains desired SLL, whereas DE maintains good directivity. On the whole this conformal shape proves much advantages because of its uniformity in tracking entire space. Hemispherical array arrangement gives narrow beam pattern for the same number of elements For very large number of elements the antenna array becomes ideal. Future work can focus on the enhancement of this arbitrary shape for medical application such as tumour treatment with the tissue exposed to controlled electromagnetic field.

\section{Appendix}

In this section, we provide the results of testing the performance of DE and FA over six representative benchmark functions from the test suite of the Congress on Evolutionary Computation (CEC) 2005 Special Session and Competition on Real Parameter Optimization [30]. All the benchmarks have been tested for dimensions $D=30$, independent runs of 
TABLE 2: Run time and fitness comparison of standard functions.

\begin{tabular}{|c|c|c|c|c|}
\hline Test function & Equation & & $\mathrm{DE}$ & FA \\
\hline Rosenbrock's & \multirow{4}{*}{$f(x, y)=(1-x)^{2}+100\left(y-x^{2}\right)^{2}$} & $\mathrm{~N}$ & 2 & 2 \\
\hline \multirow{3}{*}{ equation } & & Fitness & 0 & 0 \\
\hline & & Iterations & 1592 & 6451 \\
\hline & & CPU time (sec) & 0.1872 & 465.1170 \\
\hline \multirow[t]{4}{*}{ Griewank's } & \multirow{4}{*}{$f(x)=1+\frac{1}{400} \sum_{i=1}^{n} x_{i}^{2}-\prod_{i=1}^{n} \cos \frac{x_{i}}{\sqrt{i}}$} & $\mathrm{~N}$ & 30 & 30 \\
\hline & & Fitness & 0 & 0 \\
\hline & & Iterations & 2501 & 2664 \\
\hline & & CPU time (sec) & 0.1825 & 166.0471 \\
\hline \multirow[t]{2}{*}{ Ackley's } & \multirow{4}{*}{$\begin{array}{l}f(x)=-20 \exp ^{(-1 / 5)} \sqrt{(1 / n) \sum_{i=1}^{n} x_{i}^{2}} \\
-\exp ^{(-1 / n) \sum_{i=1}^{n} \cos 2 \pi x_{i}} 20+e\end{array}$} & $\mathrm{~N}$ & 30 & 30 \\
\hline & & Fitness & 0 & 0 \\
\hline \multirow[t]{2}{*}{ equation } & & Iterations & 5960 & 6451 \\
\hline & & CPU time (sec) & 4.3368 & 96.1170 \\
\hline Rastrigin's & \multirow{4}{*}{$f(x)=\sum_{i=1}^{n}\left(x_{i}^{2}-A \cos 2 \pi x_{i}\right)+A n$} & $\mathrm{~N}$ & 30 & 30 \\
\hline \multirow{3}{*}{ equation } & & Fitness & 0 & 0 \\
\hline & & Iterations & 9548 & 9751 \\
\hline & & CPU time (sec) & 6.9108 & 461.5791 \\
\hline
\end{tabular}

each of the algorithms were carried out, and the average and the computing time for each algorithm and the number of iterations to obtain the least fitness are noted down. Absolute run time and number of iterations of standard functions are tabulated. Table 2 is a statistical comparison on the performance of the two algorithms in terms of computing time and number of iterations required to reach a least fitness (zero). To have a fair comparison, both initial population sizes are set for the same value.

\section{Conflict of Interests}

The authors declare that there is no conflict of interests regarding the publication of this paper.

\section{References}

[1] C. A. Balanis, Antenna Theory: Analysis and Design, John Wiley \& Sons, Hoboken, NJ, USA, 3rd edition, 2012.

[2] K.-K. Yan and Y. Lu, "Sidelobe reduction in array-pattern synthesis using genetic algorithm," IEEE Transactions on Antennas and Propagation, vol. 45, no. 7, pp. 1117-1122, 1997.

[3] A. Tennant, M. Dawoud, and A. Anderson, "Array pattern nulling by element position perturbations using a genetic algorithm," Electronics Letters, vol. 30, no. 3, pp. 174-176, 1994.

[4] B. Kumar and G. Branner, "Generalized analytical technique for the synthesis of unequally spaced arrays with linear, planar, cylindrical or spherical geometry," IEEE Transactions on Antennas and Propagation, vol. 53, no. 2, pp. 621-634, 2005.

[5] J. Jin, H. Wang, W. Zhu, and Y. Liu, "Array patterns synthesizing using genetic algorithm," PIERS Online, vol. 2, no. 1, pp. 64-68, 2006.

[6] Z. Xu, H. Li, Q.-Z. Liu, and J.-Y. Li, "Pattern synthesis of conformal antenna array by the hybrid genetic algorithm," Progress in Electromagnetics Research, vol. 79, pp. 75-90, 2008.
[7] Y. Cengiz and H. Tokat, "Linear antenna array design with use of genetic, memetic and tabu search optimization algorithms," Progress in Electromagnetics Research C, vol. 1, pp. 63-72, 2008.

[8] C. Dolph, "A current distribution for broadside arrays which optimizes the relationship between beam width and side-lobe level," Proceedings of the IRE, vol. 34, no. 6, pp. 335-348, 1946.

[9] M. Skolnik, G. Nemhauser, and J. Sherman III, "Dynamic programming applied to unequally spaced arrays," IEEE Transactions on Antennas and Propagation, vol. 12, no. 1, pp. 35-43, 1964.

[10] R. Harrington, "Sidelobe reduction by nonuniform element spacing," IRE Transactions on Antennas and Propagation, vol. 9, no. 2, pp. 187-192, 1961.

[11] D. King, R. Packard, and R. Thomas, "Unequally-spaced, broad-band antenna arrays," IRE Transactions on Antennas and Propagation, vol. 8, no. 4, pp. 380-384, 1960.

[12] M. A. Panduro, D. H. Covarrubias, C. A. Brizuela, and F. R. Marante, "A multi-objective approach in the linear antenna array design," International Journal of Electronics and Communications, vol. 59, no. 4, pp. 205-212, 2005.

[13] W.-C. Weng, F. Yang, and A. Z. Elsherbeni, "Linear antenna array synthesis using Taguchi's method: a novel optimization technique in electromagnetics," IEEE Transactions on Antennas and Propagation, vol. 55, no. 3, pp. 723-730, 2007.

[14] Y.-J. Zhang, S.-X. Gong, and Y.-X. Xu, "Radiation pattern synthesis for arrays of conformal antennas mounted on an irregular curved surface using modified genetic algorithms," Journal of Electromagnetic Waves and Applications, vol. 23, no. 10, pp. 1255-1264, 2009.

[15] S. Pal, B. Qu, S. Das, and P. Suganthan, "Optimal synthesis of linear antenna arrays with multi-objective differential evolution," Progress in Electromagnetics Research B, vol. 21, pp. 87-111, 2010.

[16] Y. Rahmat-Samii and E. Michielssen, Electromagnetic Optimization by Genetic Algorithms, John Wiley \& Sons, Hoboken NJ, USA, 1999.

[17] Y.-B. Tian and J. Qian, "Improve the performance of a linear array by changing the spaces among array elements in terms 
of genetic algorithm," IEEE Transactions on Antennas and Propagation, vol. 53, no. 7, pp. 2226-2230, 2005.

[18] N. Jin and Y. Rahmat-Samii, "Advances in particle swarm optimization for antenna designs: real-number, binary, singleobjective and multiobjective implementations," IEEE Transactions on Antennas and Propagation, vol. 55, no. 3 I, pp. 556-567, 2007.

[19] Y. Chen, S. Yang, and Z. Nie, "The application of a modified differential evolution strategy to some array pattern synthesis problems," IEEE Transactions on Antennas and Propagation, vol. 56, no. 7, pp. 1919-1927, 2008.

[20] A. Mandal, H. Zafar, S. Das, and A. Vasilakos, "A modified differential evolution algorithm for shaped beam linear array antenna design," Progress in Electromagnetics Research, vol. 125, pp. 439-457, 2012.

[21] X. Li, W.-T. Li, X.-W. Shi, J. Yang, and J.-F. Yu, "Modified differential evolution algorithm for pattern synthesis of antenna arrays," Progress in Electromagnetics Research, vol. 137, pp. 371388, 2013.

[22] U. K. Chakraborty, Advances in Differential Evolution, vol. 143 of Studies in Computational Intelligence, Springer, Berlin, Germany, 2008.

[23] J.-L. Guo and J.-Y. Li, "Pattern synthesis of conformal array antenna in the presence of platform using differential evolution algorithm," IEEE Transactions on Antennas and Propagation, vol. 57, no. 9, pp. 2615-2621, 2009.

[24] A. Chowdhury, A. Ghosh, R. Giri, and S. Das, "Optimization of antenna configuration with a fitness-adaptive differential evolution algorithm," Progress in Electromagnetics Research B, vol. 26, pp. 291-319, 2010.

[25] B. Basu and G. Mahanti, "Thinning of concentric two-ring circular array antenna using fire fly algorithm," Scientia Iranica, vol. 19, no. 6, pp. 1802-1809, 2012.

[26] S. Yang, Y. B. Gan, and A. Qing, "Antenna-array pattern nulling using a differential evolution algorithm," International Journal of RF and Microwave, vol. 14, no. 1, pp. 57-63, 2004.

[27] E. Aksoy and E. Afacan, "Planar antenna pattern nulling using differential evolution algorithm," International Journal of Electronics and Communications, vol. 63, no. 2, pp. 116-122, 2009.

[28] M. A. Zaman and A. Matin, "Nonuniformly spaced linear antenna array design sing firefly algorithm," International Journal of Microwave Science and Technology, vol. 2012, Article ID 256759, 8 pages, 2012.

[29] R. Li, L. Xu, X.-W. Shi, N. Zhang, and Z.-Q. Lv, "Improved differential evolution strategy for antenna array pattern synthesis problems," Progress in Electromagnetics Research, vol. 113, pp. 429-441, 2011.

[30] P. N. Suganthan, N. Hansen, J. J. Liang et al., "Problem definitions and evaluation criteria for the cec 2005 special session on real-parameter optimization," KanGAL Report 2005005, 2005. 

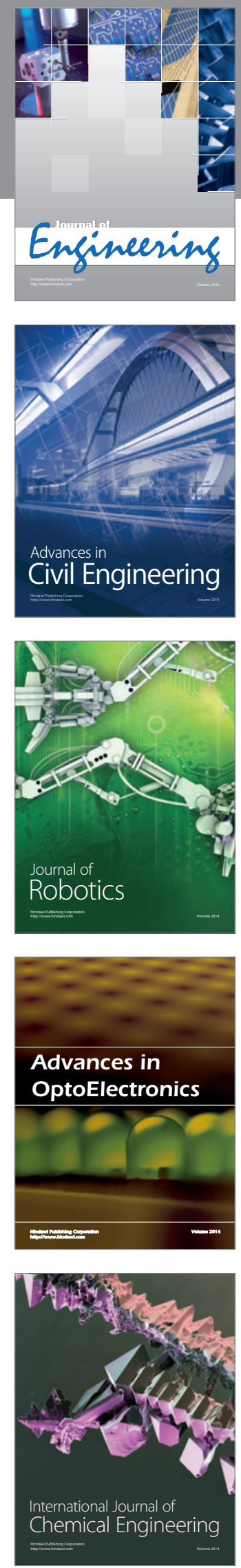

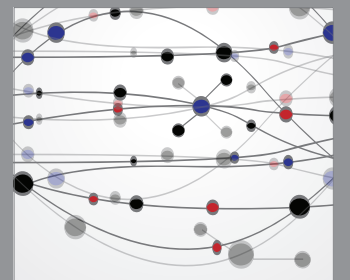

The Scientific World Journal
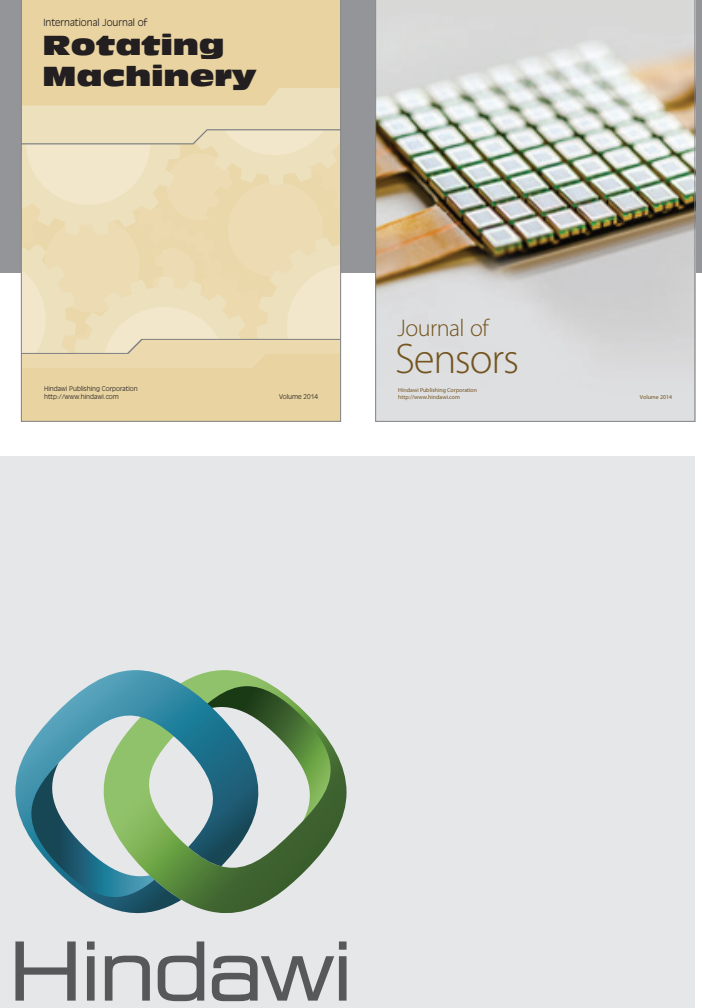

Submit your manuscripts at http://www.hindawi.com
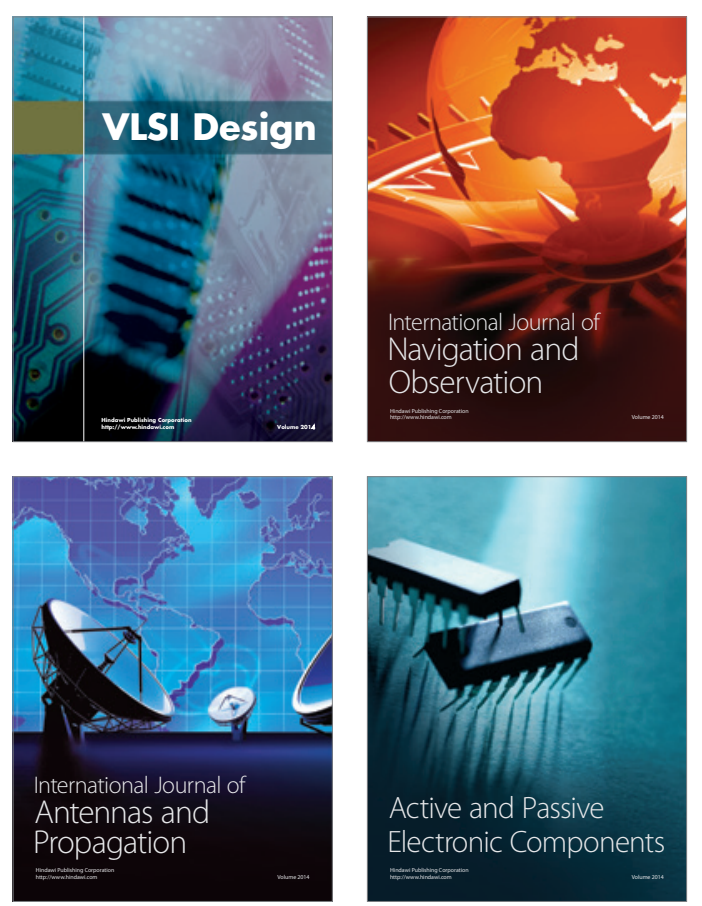
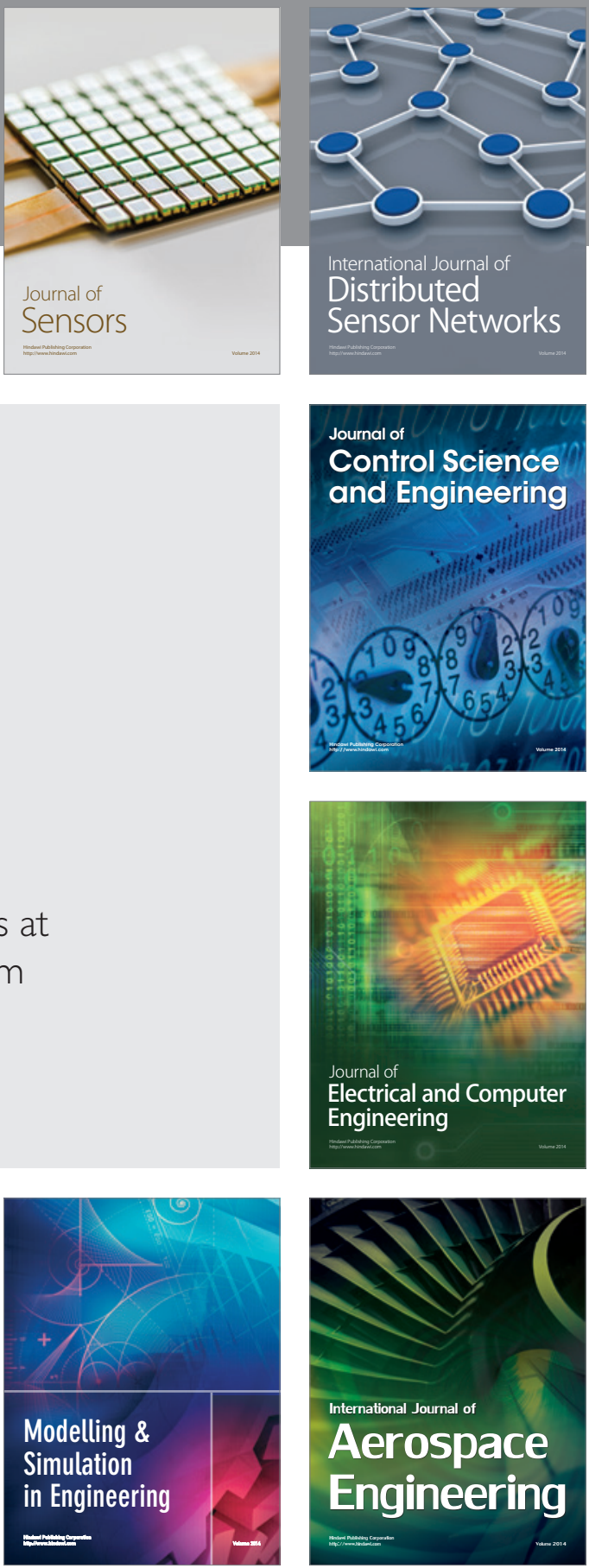

Journal of

Control Science

and Engineering
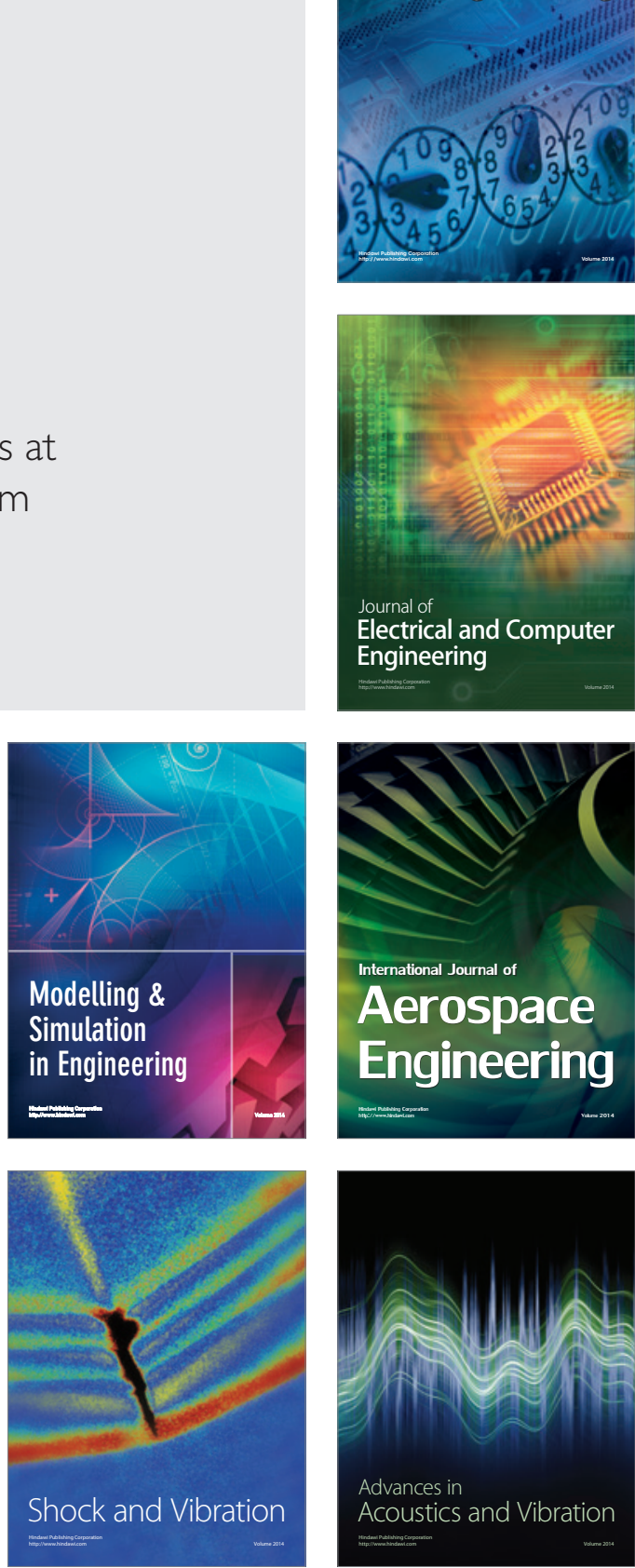